\title{
Fibroma cardíaco mimetizando cardiomiopatia hipertrófica
}

\author{
Luís Alberto DALLAN*, Sérgio de Almeida OLIVEIRA*, Antônio Carlos P. BARRETO*, José Carlos Rossini \\ IGLÉZIAS*, Geraldo VERGINELLI*, Adib D. JATENE*
}

RBCCV 44205-100

DALLAN, L. A.; OLIVEIRA, S. A.; BARRETO, A. C. P.; IGLEZIAS, J. C. R.; VERGINELLI, G.; JATENE, A. D. - Fibroma cardiaco mimetizando cardiomiopatia hipertrófica. Rev. Bras. Cir. Cardiovasc., 4(3): 242-248, 1989.

RESUMO: É relatado o caso de paciente com queixa de dor precordial. dispnéia e arritmia desde a adolescência, tratada clinicamente por mais de 10 anos. Nesse periodo, foi submetida a inúmeros exames ângio e ecocardiográficos, com suspeita inicial de endomiocardiofibrose e, posteriormente, de cardiomiopatia hipertrófica de ventrículo esquerdo. Como houve piora progressiva da sintomatologia e ausência de resposta à medicaçāo, foi encaminhada ao nosso Serviço, onde se diagnosticou fibroma de ventrículo esquerdo. Foi submetida, com sucesso, à ressecção cirúrgica do tumor, sendo realizada reconstrução geométrica do ventrículo esquerdo. Apresenta boa evolução, decorridos dois anos, com remissão completa dos sintomas. Destacamos a dificuldade no diagnóstico diferencial desses tumores benignos e de crescimento lento, com as cardiomiopatias hipertróficas do ventrículo esquerdo.

DESCRITORES: tumores do coração; miocardiopatia hipertrófica.

\section{INTRODUÇĀO}

Os tumores primários do coraçāo são raros ${ }^{4}$ e,até há poucas décadas, constituiam, em sua maioria, achados de necropsia ${ }^{21}$. A evolução da propedêutica médica e dos métodos diagnósticos, especialmente os não invasivos, vêm possibilitando a identificaçāo e o tratamento adequado de inúmeros tumores cardíacos. Entretanto, os sintomas desses tumores, invariavelmente, são inespecíficos e dependem do grau de obstrução que seu crescimento proporciona à cavidade em que se localizam. A expansão lenta de alguns deles pode dificultar seu diagnóstico precoce, ou mesmo mimetizar sinais e sintomas de outras afecçōes cardiacas, cujo tratamento é totalmente distinto.

Relatamos o caso de paciente portadora de fibroma de ventrículo esquerdo, cujo diagnóstico foi confundido com miocardiopatia hipertrófica por mais de 10 anos, apesar de ter acompanhamento eco e cineangiográfico em diferentes centros de Cardiologia.

\section{RELATO DO CASO}

Trata-se de paciente do sexo feminino, 33 anos, com queixa, desde a adolescência, de dispnéia e cansaço aos médios esforços, associados a dor precordial em opressão, por 15 minutos, com regressão espontânea.

Há 11 anos, durante consulta, apresentou palpitações e cianose, seguidas de parada cardiorespiratória, sendo reanimada, com sucesso. Nessa época, foi submetida a cateterismo cardiaco, que revelou falha de enchimento no ventrículo esquerdo (VE), tendo sido estabelecido o diagnóstico de endomiocardiofibrose (EMF)

Trabalho realizado no Instituto do Coração do Hospital das Clinicas da Faculdade de Medicina da Universidade de São Paulo, São Paulo, SP. Brasil.

Recebido para publicaçâo em 30 de dezembro de 1989.

-Do Instituto do Coração do Hospital das Clinicas da Faculdade de Medicina da Universidade de São Paulo.

Endereço para separatas: Luis Alberto Dallan. Av. Dr. Enéas Carvalho de Aguiar, 44. Divisão Cirúrgica. 05403 São Paulo, SP, Brasil. 
DALLAN, L. A.; OLIVEIRA, S. A.; BARRETO, A. C. P.; IGLEZIAS, J. C. R.; VERGINELLI, G.; JATENE, A. D. - Fibroma cardiaco mimetizando cardiomiopatia hipertrófica. Rev. Bras. Cir. Cardiovasc., 4(3): 242-248, 1989.

de VE. Foi medicada com antiarrítmicos por seis anos, período em que se intensificou a dispnéia e desenvolveu edema de membros inferiores. Transferida para outro Serviço de Cardiologia, foi submetida a exames ecocardiográficos, tendo-se suspeitado de cardiomiopatia hipertrófica. Apesar de receber altas doses de betabloqueadores e de antiarrítmicos, houve piora progressiva da sintomatologia. Passou a apresentar dispnéia paroxística noturna e episódios sincopais freqüentes, tendo procurado o Instituto do Coração.

Como principais antecedentes, destacam-se: amigdalectomia, cirurgia do septo nasal, fraturas de membro superior esquerdo e inferior direito e exérese de cisto de ovário direito, aos 17 anos. Não há relato de sintomas semelhantes em nenhum de seus familiares.

Ao exame físico, apresentava-se em bom estado geral, eutrófica, corada. O pulso era palpável em todos os membros, com freqüência média de 100 batimentos por minuto e raras extra-sístoles. A pressão arterial medida em ambos os membros superiores era de $100 \mathrm{x}$ $60 \mathrm{mmHg}$. A ausculta torácica revelava murmúrio vesicular presente em ambos os hemitóraces, sem ruídos adventícios. O ictus cardíaco era visível no $5^{\circ}$ espaço intercostal esquerdo, sem frêmitos. As bulhas cardiacas encontravam-se discretamente hipofonéticas, sem sopros. O abdômen apresentava-se flácido, sem hepato ou esplenomegalia. Os ruídos hidroaéreos estavam presentes e normais. Notava-se edema moderado de membros inferiores.

Os exames de sangue no pré-operatório encontravam-se dentro dos limites da normalidade, com exceção de discreta anemia.

O eletrocardiograma demonstrava ritmo sinusal com extra-sístoles ventriculares, área inativa septal e alterações da repolarização ventricular. Os exames ecocardiográficos revelavam fluxos transvalvares normais, diminuiçāo da cavidade ventricular esquerda e hipocinesia da região apical da parede ântero-septal, com fraçăo de ejeção de 0,69.

Holter instalado durante 24 horas detectou 545 extra-sístoles ventriculares polimórficas, de ocorrência aleatória. A conduções atrioventriculares (PR $=0,16$ seg.) e interventriculares (QRS $=0,08$ seg.) não mostraram modificações significativas.

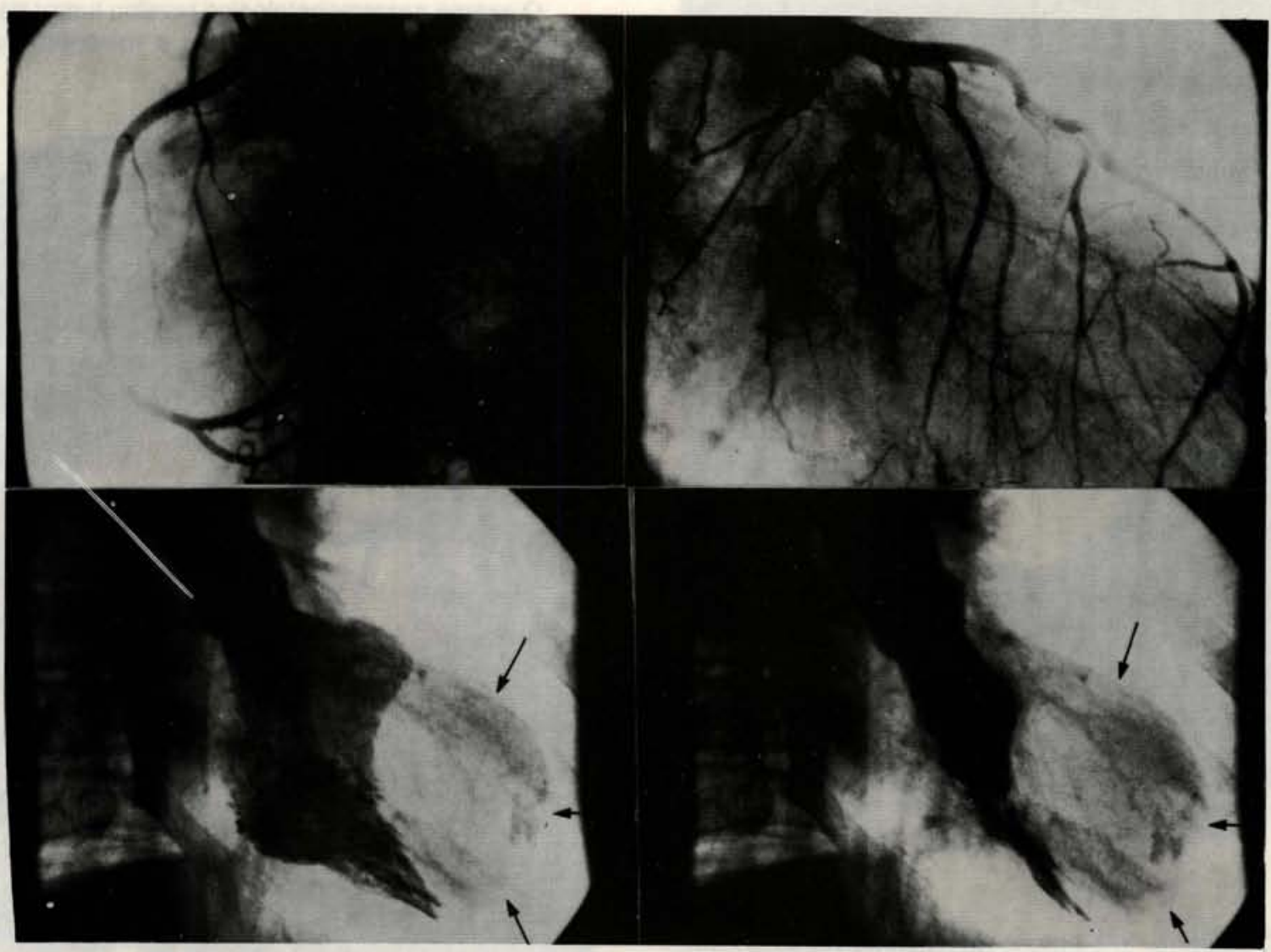

Fig. 1 - Superior: cinecoronariografia D e E, mostrando artérias coronárias de bom calibre e sem lesర̄es obstrutivas. Inferior: venticulografia $\mathrm{E}$ em diástole e sistole, com falha de enchimento em regiōes ântero-apical e lateral (setas), devido a fibroma intramural de VE. 
DALLAN, L. A.; OLIVEIRA, S. A.; BARRETO, A. C. P.; IGLEZIAS, J. C. R.; VERGINELLI, G.; JATENE, A. D. - Fibroma cardiaco mimetizando cardiomiopatia hipertrófica. Rev. Bras. Cir. Cardiovasc., 4(3): 242-248, 1989.

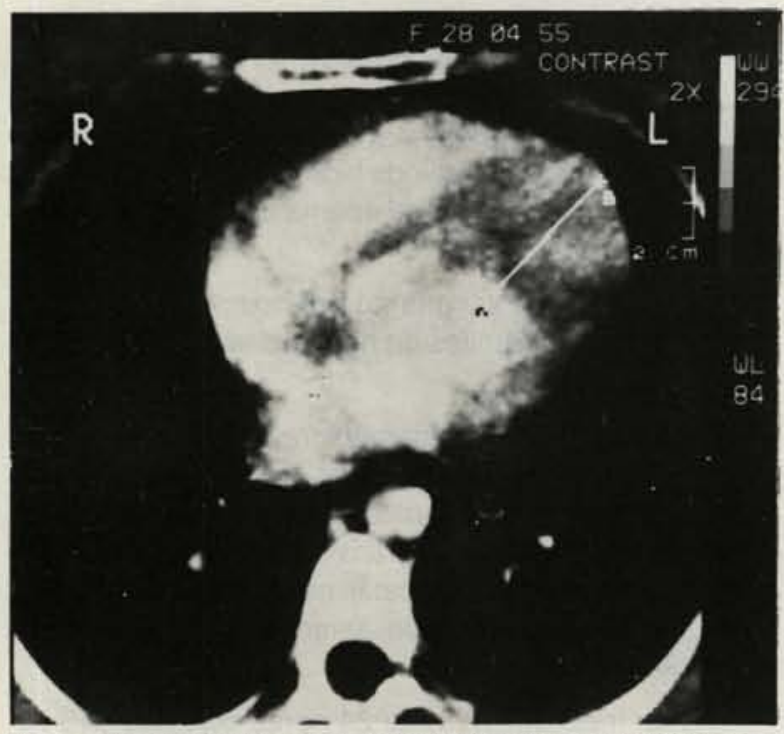

Fig. 2 - Tomografia computadorizada axial do tórax: são evidenciadas as paredes septal e lateral do VE (mais contrastadas) e área menos densa, correspondente à região ântero-septal comprometida pelo fibroma.

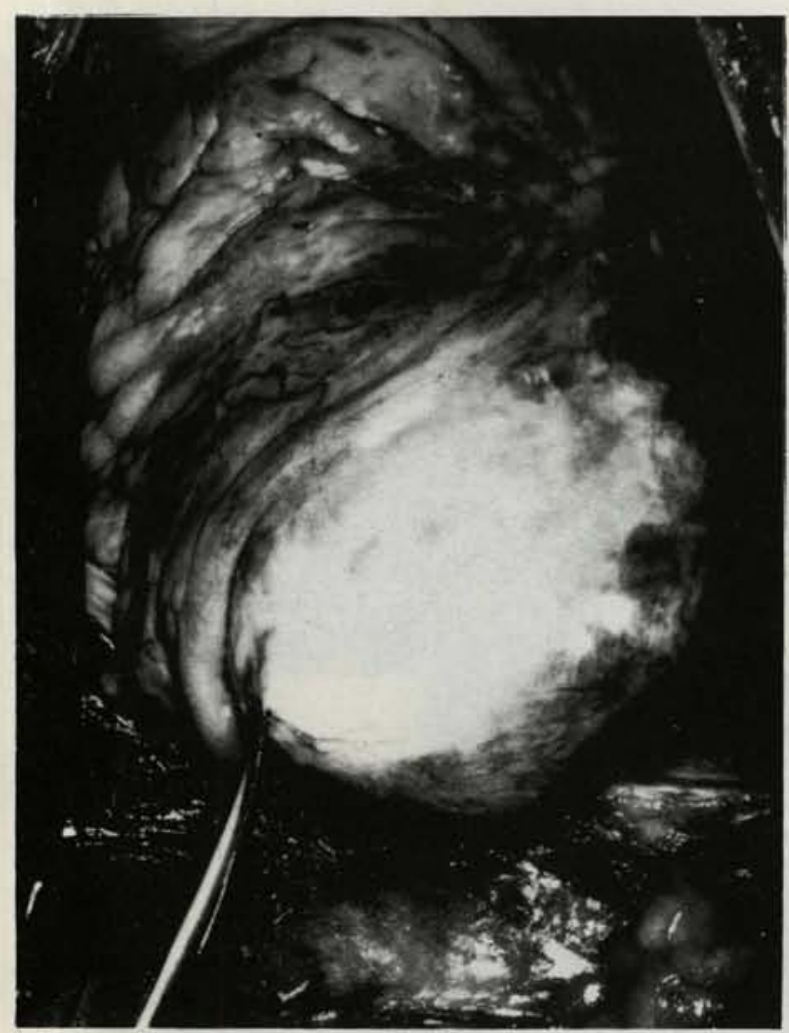

Fig. 3 - Aspecto externo do VE, destacando-se massa tumoral de aspecto em meio à região ântero-apical e lateral do VE.

O cateterismo cardíaco demonstrou pressōes normais nas cavidades cardiacas direitas e esquerdas. A circulação coronária nāo apresentava lesōes obstrutivas. A ventriculografia, entretanto, revelou redução acentua- da da cavidade esquerda às custas de massa não contrátil, que ocupa sua porção ântero-septal, estendendo-se até a parede lateral (Figura 1). Aspecto semelhante foi observado em tomografia computadorizada (Figura 2).

A paciente foi submetida a cirurgia com o auxílio da circulaçāo extracorpórea, hipotermia moderada e solução cardioplégica. A inspeçāo cardíaca revelou massa tumoral de consistência dura e easpecto nacarado comprometendo a parede ântero-septal do VE, extendendo-se até sua face lateral (Figura 3). O VE foi incisado em seu eixo longitudinal, paralelamente à artéria descendente anterior (Figura 4). O tumor media $9 \times 6 \times 5 \mathrm{~cm}$, não era irrigado e localizava-se em meio à parede do VE, atingindo as proximidades do endocárdio. Sua ressecção foi realizada cuidadosamente, tendo-se preservado as artérias coronárias e os músculos papilares (Figura 2). A abertura da cavidade do VE visou ampliar a margem da ressecção tumoral. O VE foi reconstruído com o auxílio de retalho de pericárdio bovino com 3 $\mathrm{cm}$ de diâmetro (Figura 6) sobre a qual foram suturadas as bordas remanescentes do VE. A retomada da função cardiaca ocorreu sem problemas, com ritmo sinusal. rências.

O pós-operatório imediato transcorreu sem intercor-

O exame anatomopatológico revelou tumor de conformação nodular pesando $110 \mathrm{gr}$ e medindo $9 \times 6,5$

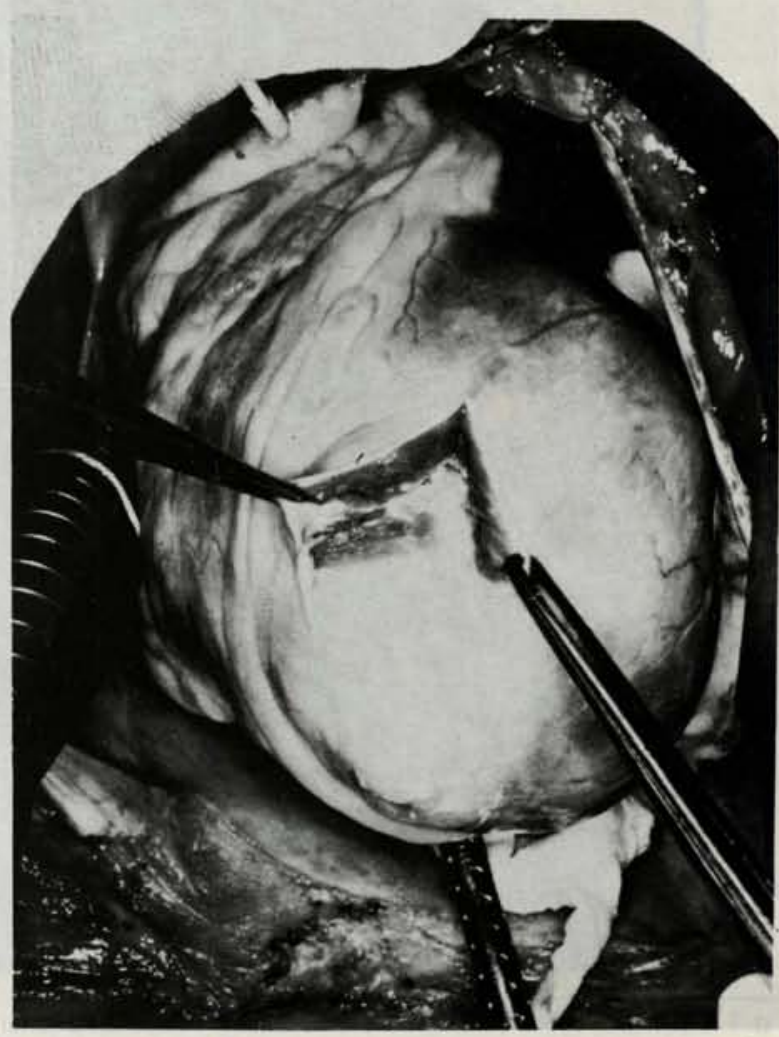

Fig. 4 - Incisão de VE paralelamente à artéria descendente anterior iniciando-se a dissecção do tumor. 
DALLAN, L. A.; OLIVEIRA, S. A.; BARRETO, A. C. P.; IGLEZIAS, J. C. R.; VERGINELLI, G.; JATENE, A. D. - Fibroma cardiaco mimetizando cardiomiopatia hipertrófica. Rev. Bras. Cir. Cardiovasc., 4(3): 242-248, 1989.

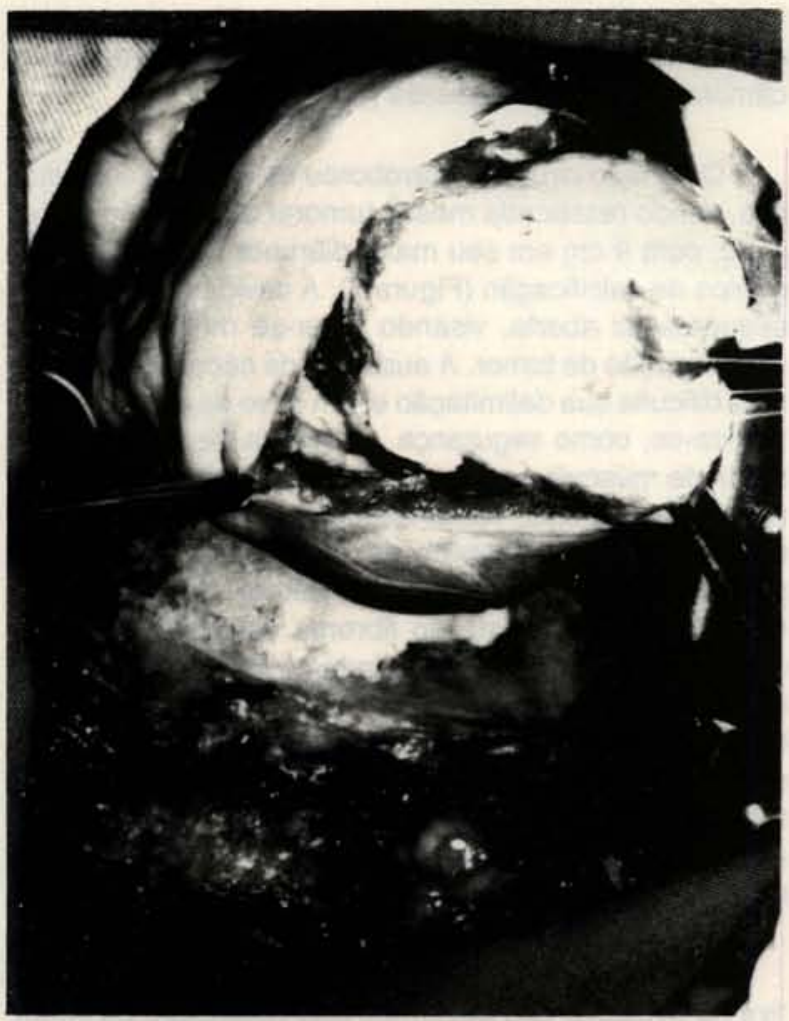

Fig. 5 - Retirada do tumor após sua dissecção cuidadosa, preservando-se as artérias coronárias e os músculos papilares da valva mitral.

$\mathrm{cm}$. Apresentava superície externa lobulada, irregular, amarelada, com áreas pardacentas (Figura 7). Aos cortes, notava-se superfície fasciculada e esbranquiçada, com consistência firme e áreas de calcificação. O exame histológico demonstrou tecido formado por bandas de colágeno e células fibroblásticas de aspecto benigno, raramente entremeado por fibras musculares cardiacas, caracterizando o fibroma cardiaco.

\section{COMENTÁRIOS}

O fibroma cardiaco é tumor benigno e raro, com incidência maior em lactentes e crianças ${ }^{1,18,22}$. Esse fato sugeriu sua provável origem congênita, embora não exista relato de sua incidência familiar ${ }^{10}$. Histopatologicamente, é lesão benigna composta por fibroblastos entremeados por fibras musculares cardíacas e bandas de colágeno ${ }^{12}$. Ocorre com maior freqüência na parede livre do ventrículo esquerdo (VE) ou septo interventricular (SIV) e os sintomas decorrem de sua interferência mecânica com o fluxo intracardíaco, contração ventricular ou distúrbio de condução ${ }^{5}$. A manifestação clínica, se traduz, em geral, por sopros, insuficiência cardiaca congestiva, sinais de estenose subaórtica, associados ou nāo à dor torácica atípica. O seu caráter benigno e crescimento lento não são incompatíveis com uma maior longe-

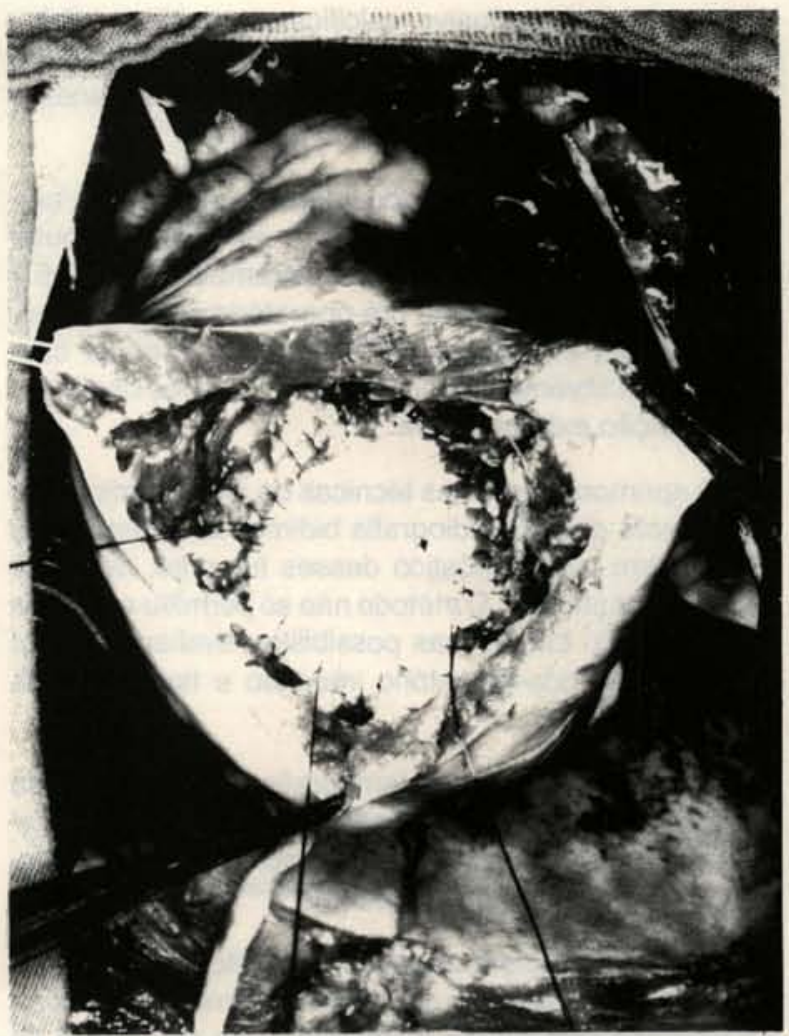

Fig. 6 - Retalho de pericárdio bovino usado na reconstrução geométrica da cavidade ventricular. $E$, sobre o qual foram suturadas as bordas remanescentes do VE.

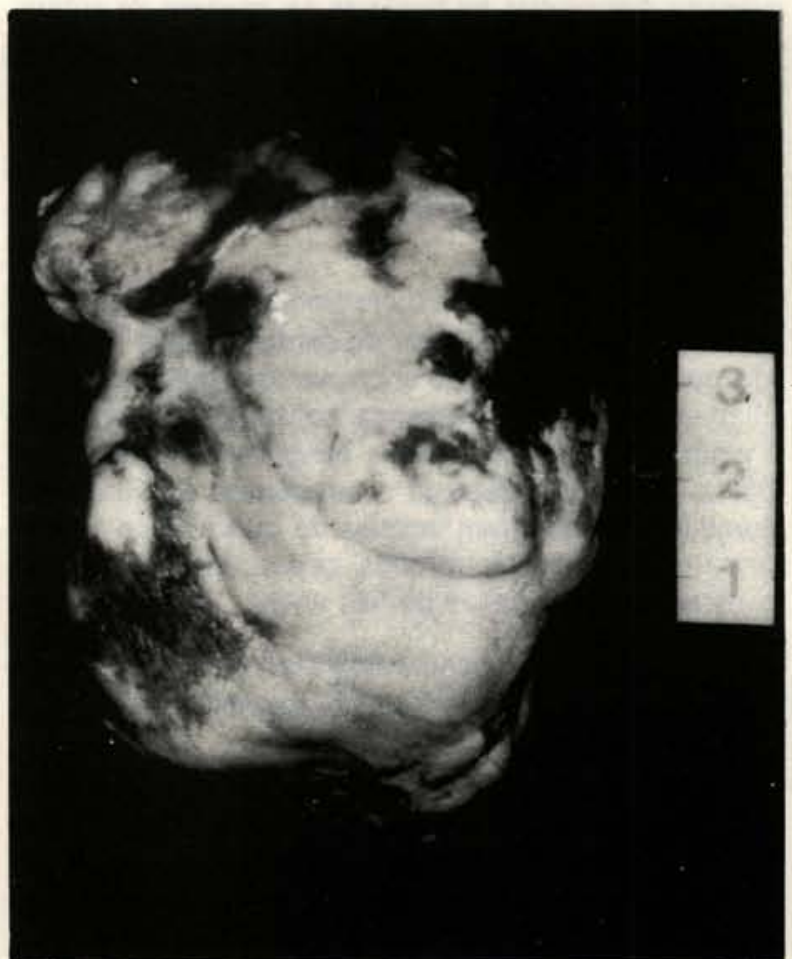

Fig. 7 - Aspecto macroscópico do fibroma, com superticie externa lobulada e irregular. 
vidade, podendo, inclusive, calcificar-se na idade adulta. Entretanto, a morte súbita e inesperada constitui uma importante complicaçāo do tumor, e traduz a necessidade de diagnóstico e tratamento precoses ${ }^{13}$.

O primeiro caso bem documentado de fibroma cardíaco foi relatado por LUSCHKA ${ }^{17}$, em 1885 , e coube a EDLUNG \& HOLMDAHL ${ }^{7}$ pioneiramente, em 1957 , diagnosticarem o tumor no pré-operatório de um paciente. Em 1962, PARKS et alii ${ }^{19}$ lograram remover com sucesso um fibroma da parede do VE, através do auxilio da circulação extracorpórea.

O aprimoramento nas técnicas de angiocardiografia e $o$ advento da ecocardiografia bidimensional em muito contribuíram no diagnóstico desses tumores, especialmente em crianças ${ }^{2}$. O método não só permitiu a perfeita localização do tumor, mas possibilitou avaliar a função ventricular no pós-operatório imediato e tardio desses pacientes ${ }^{8}$.

Mais recentemente, a tomografia computadorizada e a ressonância nuclear magnética vêm fornecendo imagens altamente precisas desses tumores e de suas relaçōes com as estruturas adjacentes ${ }^{9,20}$.

A despeito desses avanços nos métodos de investigação, algumas vezes o diagnóstico diferencial do fibroma cardiaco com outras miocardiopatias não é fácil de se estabelecer. Esta afirmaçāo pode ser bem verificada no caso relatado. $O$ diagnóstico inicial atribuído à paciente, em outro Serviço, foi endomiocardiofibrose de VE, estabelecido em estudo angiográfico. Possivelmente, a suspeita diagnóstica foi baseada na imagem de subtração da cavidade ventricular, observada na ventriculografia esquerda. Entretanto, não foi considerada a ausência de retraçāo da ponta do VE e o não comprometimento dos músculos papilares da valva mitral, que, freqüentemente, estão presentes na endomiocardiofibrose de VE.

Ao transferir-se para outro Serviço, decorridos seis anos, passou-se a suspeitar de cardiomiopatia hipertrófica, dada a dificuldade de enchimento da câmara ventricular esquerda, e a presença constante de arritmias. A piora gradativa da dispnéia atingindo o repouso e a total ausência de resposta à terapêutica com betabloqueadores constituíram indícios de que não se tratava de cardiomiopatia hipertrófica, o que, entretanto, não foi considerado por mais de três anos.

Estudos ângio e ecocardiográfico realizados posteriormente, no Instituto do Coraçāo, permitiram identificar tumor localizado e de grandes proporçōes em meio à parede livre do VE, restringindo, significativamente, a câmara ventricular esquerda (Figura 1).

O achado cirúrgico corroborou os exames subsidiários, sendo ressecada massa tumoral consistente e irregular, com $9 \mathrm{~cm}$ em seu maior diâmetro, já com alguns pontos de calcificação (Figura 7). A cavidade ventricular esquerda foi aberta, visando obter-se melhor margem de ressecção do tumor. A ausência de cápsula nos fibromas dificulta sua delimitação e, em caso de dúvida, recomenda-se, como segurança, a retirada de pequena camada de músculo saudável junto do mesmo ${ }^{20}$. O fechamento da cavidade remanescente do VE foi realizado com o emprego de retalho de pericárdio bovino, o que possibilitou a reconstrução geométrica do VE (Figura 6). A localização exata do fibroma deve ser estudada com detalhes pelo cirurgião ainda no pré-operatório, antenvendo eventuais reparos nas estruturas comprometidas. Como grande percentagem dos fibromas incide sobre o septo interventricular, sua ressecção pode resultar em comunicação entre os ventrículos, ou em danos ao sistema de condução, e o cirurgião deve estar preparado para o tratamento dessas complicações ${ }^{20}$.

Os relatos da literatura revelam excelente perspectiva na evoluçāo dos pacientes cuja ressecção do tumor cardíaco benigno foi considerada completa ${ }^{6}$. Entretanto, é recomendável o exame ecocardiográfico periódico, visando detectar raríssimas recidivas ${ }^{21}$. Bons resultados tardios também têm sido descritos em pacientes cuja ressecção da massa tumoral foi apenas parcial e não receberam terapêutica complementar ${ }^{24}$, inclusive com desaparecimento dos sintomas de insuficiência cardíaca congestiva ${ }^{11,}{ }^{16}$. Em pacientes cujos estados evolutivos do fibroma não permitiram sequer sua ressecção parcial, o transplante cardíaco foi empregado como última alternativa ${ }^{15}$. Nossa paciente vem apresentando boa evolução pós-operatória, decorridos mais de dois anos. Seguimento clínico e ecocardiográfico revelaram normalização da contratilidade de VE, desaparecimento da dispnéia e da arritmia e regressão de classe funcional IV para I.

Acreditamos que a demora na elucidação diagnóstica do presente caso decorreu não só da raridade desse tipo de tumor, mas especialmente por sua extensão e crescimento lento, mimetizando cardiomiopatia hipertrófica. Entretanto, em casos semelhantes, deverá, de inicio, ser considerada a possibilidade de tratar-se de fibroma cardíaco. 
DALLAN, L. A.; OliVEIRA, S. A.; BARRETO, A. C. P.; IGLEZIAS, J. C. R.; VERGINELLI, G.; JATENE, A. D. - Cardiac fibroma mimicking hypertrophic cardiomyopathy.. Rev. Bras. Cir. Cardiovasc., 4(3): 242-248, 1989.

ABSTRACT: A 33 year-old woman was seen, for the first time, ten years ago, for evaluation of a recurrent chest pain, dyspnea and arrhythmia. She was submitted to echocardiographic studies and a cardiac catheterization. The diagnoses was endomyocardial fibrosis at first, and hypertrophic cardiomyopathy after. Despite treatment with propranolol and quinidine, the episodes of dyspnea and tachyarrhythmias became more frequent and severe, and the patient was guided to our Service. Cardiac re-catheterization, echocardiographic and computed tomography studies identified in traumural cardiac fibroma and the patient was referred for surgical treatment. The cardiac fibroma was successfully resected on extracorporeal bypass and with cardioplegic arrest of the heart. Repair of the heart was accomplished with a patch placed to close the left ventricular cavity. The postoperative course was uncomplicated, and she remains assymptomatic two years later. We have emphazied tha this tumor often produces clinically obscure disease, simulating particularly the left ventricle hypertrophic cardiomiopathy.

DESCRIPTORS: tumors of the heart; cardiomiopathy, hypertrophic.

\section{REFERÊNCIAS BIBLIOGRÁFICAS}

1 ARCINIEGAS, E.; HAKINI, M.; FAROOKI, Z. Q.; TRUCCONE, N. J.; GREEN, E. W. - Primary cardiac tumors in children. J. Thorac. Cardiovasc. Surg., 79: 582-591, 1980.

2 BIANCANIELLO, T. M.; MEYER, R. A.; GAUM, W. E.; KAPLAN, S. - Primary benign intramural ventricular rumors in children: pre-and postoperative electrocardiographic, echocardiographic, and angiocardiographic evolution. Am. Heart J., 103: 852-857, 1982.

3 CABIN, H. S.; COSTELLO, R. M.; VASUDEVAN, G.; MARON, B. J.; ROBERTS, W. C. - Cardiac lymphome mimicking hypertrophic cardiomyopathy. Am. Heart J., 102: 466-468, 1981.

4 CHITWOOD, W. R. - Cardiac neoplasms: current diagnosis, pathology, and therapy. J. Cardiac. Surg., 3: 119-154, 1988.

5 DALLAN, L. A. O. - Tumores do coração. In: RAIA, A. A. \& ZERBINI, E. J. eds. Clinica cirúrgica Alipio Corrêa Neto. São Paulo, Sarvier, 1988. p.

6 DEIN, J. R.; FRIST, W. H.; STINSON, E. B.; MILLER, C.; BALDWIN, J. C.; OYER, P. E.; JAMIESON, S.; MITCHELL, R. S.; SHYMWAY, N. E. - Primary cardiac neoplsms: early and late results of surgical treatment in 42 patients. J. Thorac. Cardiovasc. Surg., 93: 502-511, 1987.

7 EDLUND, S. \& HOLMDAHL, K. - Primary tumor of the heart: report of a case. Acta Paediat., 46: 59-63, 1957.

8 ETCHES, P. C.; GRIBBIN, B.; GUANNING, A. J. - Echocardiographic diagnosis and successful removal of cardiac fibroma in a four-year-old child. Br. Heart $J$. , 43: 360-362, 1980.
9 FREEDBERG, R. S.; KRONZON, I.; RUMANCIC, W. M.; LIEBESKIND, L. - The contribution of magnetic resonance imaging to the evaluation of intracardiac tumors diagnosed by echocardiography. Circulation, 77: 96-103, 1988.

10 GEHA, A. S.; WIEDMAN, W. H.; SOULE, E. H.; McGOON, D. C. - Intramural ventricular fibroma: successful removal in two cases and review of the literature. Circulation, 36: 427-440,

11 HARDIM, N. J.; WILSON, S. M.; GRAY, G. F.; GAY, W. A. - Experience with primary tumors of the heart: clini$\mathrm{cal}$ and pathological study of seventeen cases. John Hopkins Med. J., 134: 141-155, 1974.

12 HEATH, D. - Pathology of cardiac tumors. Am. J. Cardiol., 21: 315-327, 1968.

13 HOEN, A. G. \& ELLIS, E. J. - Intramural fibroma of the heart. Am J. Cardiol., 17: 579-584, 1966.

14 ISNER, J. M.; FAOCONE, M. W.; VIRMANI, R.; ROBERTS, W. C. - Cardiac sarcoma causing "ASH" and simulating coronary heart disease. Am. J. Med., 66: 1025-1030, 1979.

15 JAMIESON, S. W.; GAUDIANI, V. A.; EWITZ, B. A.; OYER, P. E.; STINSON, E. B.; SHUMWAY, N. E. - Operative treatment of an unresectable tumor of the left ventricle. J. Thorac. Cardiovasc. Surg., 81: 797-799, 1981.

16 LINCOLN, J. C. R.; TYNAM, M. G.; WATERSTON, D. J. - Successful excision of an endocardial fibroma of the left ventricle in a 10-month-old infant. J. Thorac. Cardiovasc. Surg., 56: 63-70, 1968.

17 LUSCHKA, H. - Ein fibroid im HERZ fleische. Wirchows Arch. Pathol. Anat., 8: 434, 1885.

18 NADAS, A. S. \& ELLISON, R. C. - Cardiac tumors in infancy. Am. J. Cardiol., 2: 363-366, 1968. 
DALLAN, L. A.; OLIVEIRA, S. A.; BARRETO, A. C. P.; IGLEZIAS, J. C. R.; VERGINELLI, G.; JATENE, A. D. - Fibroma cardiaco mimetizando cardiomiopatia hipertrófica. Rev. Bras. Cir. Cardiovasc., 4(3): 242-248, 1989.

19 PARKS, F. R.; ADAMS, F.; LONGMISE Jr., W. P. - Successful excision of a left ventricular hemartoma. Circulation, 26: 1316-1320, 1962.

20 PARMLEY, L. F.; SALLEY, R. K.; WILLIAMS, J. P.; HEAD, G. B. - The clinical spectrum of cardiac fibroma with diagnostic and surgical considerations noninvasive imaging enhances management. Ann. Thorac. Surg., 45: $455-465,1988$.

21 TURI, G. K.; ALBALA, A.; FENOGLIO Jr., J. J. - Cardiac fibromatosis: an ultrastructural study. Hum. Pathol., 2 (Supl.): 577-580, 1980.
22 VAN DER HAUWAERT, L. G.; CORBEEL, L.; MALDAGUE, P. - Fibroma of the right ventricle producing severe tricuspid stenosis. Circulation, 32: 451-456, 1965.

23 YABEK, S. M.; JONES, J. I.; GYEPES, M. T.; JARMAKANI, J. M. - Cardiac fibroma in a neonate presenting with severe congestive heart feilure. J. Pediatr., 91: 310-312, 1977.

24 ZERKOWSKI, H. R.; HENTRICH, F.; DOETSCH, N. W.; ROHM, N. - Partial replacement of the left ventricular wall for a large intramural fibroma. Pediatr. Cardiol., 6: $43-45,1985$. 